\title{
Automated Sensing System for Monitoring Road Surface Condition Using Fog Computing
}

\author{
Nafras Ameer B.A, Pradeep Kumar J, Kumaran V, Dinesh P, Mr. M. Dilli Babu, M.E.
}

Department of Information Technology, Panimalar Engineering College, Chennai, India

\begin{abstract}
The principle point of this task is to build up an Intelligent Monitoring System used to screen the Road Surface Condition using Fog Computing that increases the road safety. Multiple solutions have been proposed which make use of mobile sensing, more specifically contemporary applications and architectures that are used in both crowd sensing and vehicle based sensing. Nonetheless, these initiatives have not been without challenges that range from mobility support, location awareness, low latency as well as geo-distribution. As a result, a new term has been coined for this novel paradigm, called, fog computing.
\end{abstract}

Keywords-Certificateless aggregate signcryption (CLASC), fog computing, road surface condition monitoring system, security.

\section{INTRODUCTION}

\subsection{Overview of the Project}

Great attention has been directed toward road surface condition monitoring in the recent past. As a matter of fact, this activity is of critical importance in transportation infrastructure management. In response, multiple solutions have been proposed which make use of mobile sensing, more specifically contemporary applications and architectures that are used in both crowd sensing and vehicle-based sensing. This has allowed for automated control as well as analysis of road surface quality. These innovations have thus encouraged and showed the importance of cloud to provide reliable transport services to clients. Nonetheless, these initiatives have not been without challenges that range from mobility support, locational awareness, low latency, as well as geodistribution. As a result, a new term has been coined for this novel paradigm, called, fog computing. In this paper, we propose a privacy-preserving protocol for enhancing security in vehicular crowd sensing based road surface condition monitoring system using fog computing. At the onset, this paper proposes a certificate less aggregate signcryption scheme that is highly efficient.

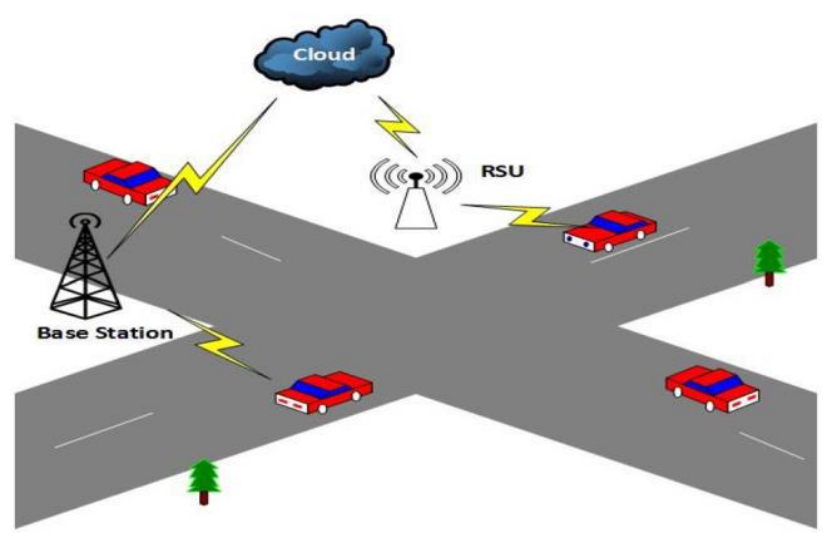

Fig.1: Cloud-based Architecture

On the basis of the proposed scheme, a data transmission protocol for monitoring road surface conditions is designed with security aspects such as information confidentiality, mutual authenticity, integrity, privacy, as well as anonymity. In analyzing the system, the ability of the proposed protocol to achieve the set objectives and exercise higher efficiency with respect to computational and communication abilities in comparison to existing systems is also considered.

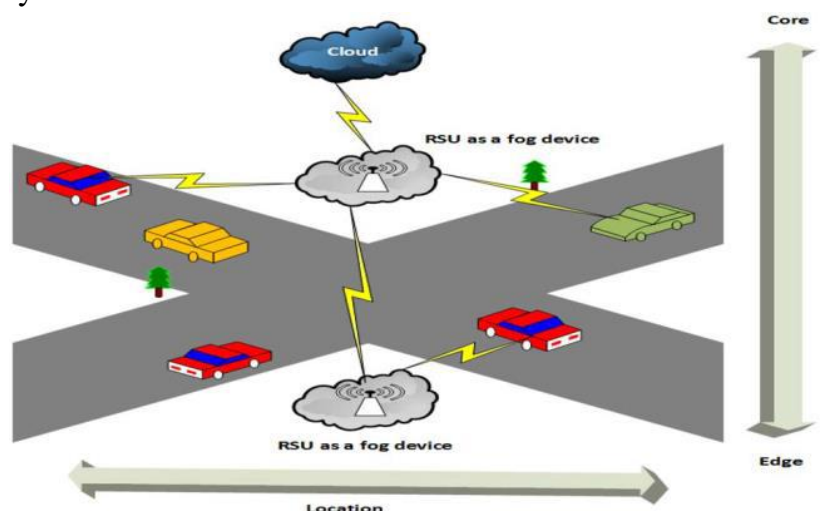

Fig.2: Fog-based Architecture

\subsection{Existing System}

The condition of road surfaces is considered as a major indicator of the quality of roads. As a matter of fact, classification of a road as either safe or dangerous, more often than not take into consideration the surface condition of the road. Conventionally, parameters such as potholes, bumps and slipperiness are considered as the distinguishing features of the quality of road surfaces. 
Thus systems for monitoring road conditions are critical to the improvement of safety in roads, lowering accident rates and protection of vehicles from getting damaged as a result of poor surface road conditions. Using advanced vehicular technologies especially vehicular communication combined with sensing technologies, road anomalies can be easily identified and dealt with. This is achieved using an advanced system for monitoring road surface condition.

\subsection{Proposed System}

We propose a Road surface Condition Monitoring System Using Fog Computing which consists of traditional OBU(On board Unit) and RSU as Fog Node. In General OBU is Responsible to monitor the road condition and forward the content to the cloud. While uploading the information to the cloud OBU must encrypt the information by using Signcryption. Control center is responsible for key generation to the OBU as well as rsu . OBU will encrypt the information by using the key provided by the control center and forward the information to the Fog cloud i.e.(RSU). Fog Node is Responsible to Verifying the truthfulness of an message and forward the information to the cloud. The Cloud will maintain the data whenever request received cloud process the data and provide the corresponding request.

\section{PROPOSED ALGORITHM AND EQUATIONS}

The proposed CLASC scheme is composed by the following six algorithms.

\subsection{Setup}

Given the security parameters $k$, and this algorithm is performed by the $\mathrm{KGC}$ as follows.

a) Chooses a cyclic additive group $G$ of prime order $q$ on elliptic curve, and $P$ is an arbitrary generator of $G$.

b) Chooses a cyclic multiplicative group $G_{\mathrm{T}}$ of the same order $q$ and a bilinear map ê: $G \times G \rightarrow G_{\mathrm{T}}$.

c) Randomly selects a master private key $s \in Z_{q}^{*}$ and compute the master public key $P_{\text {pub }}=s P$.

d) Selects four secure hash functions $H_{1}:\{0,1\}^{*} \rightarrow$ $Z_{q}^{*}, H_{2}:\{0,1\}^{*} \rightarrow\{0,1\}^{n}$ here $n$ is the bit-length of plaintexts, $H_{3}:\{0,1\}^{*} \rightarrow G$ and $H_{4}: Z_{q}^{*} \rightarrow G$.

e) Publishes the system parameter params $=$ $\left(G, G_{\mathrm{T}}, \hat{\mathrm{e}}, P, q, P_{\mathrm{pub}}, H_{1}, H_{2}, H_{3}, H_{4}\right)$ and the master private key $s$ will be kept secure by the KGC.

\subsection{Key-Generation}

This algorithm is interactively performed by the user $\mathrm{ID}_{i}$ and $\mathrm{KGC}$ as follows.

a) The user $\operatorname{ID}_{i}$ randomly chooses $x_{i} \in Z_{q}^{*}$ as the secret value and computes a partial public key $Y_{i b}=x_{i} P$.

b) The user sends its identity and partial public key
$\left(\mathrm{ID}_{i}, Y_{i b}\right)$ to the KGC.

c) The KGC then randomly selects $y_{i} \in Z_{q}^{*}$ and compute another partial public key for the user $Y_{i a}=y_{i} P$, so the full public key for the user is $\left(Y_{i b}, Y_{i a}\right)$.

d) The KGC computes the partial private key $D_{i}=$ $y_{i}+s * Q_{i}$ where $Q_{i}=H_{1}\left(\mathrm{ID}_{i}\right)$, and $D_{i}$ is sent securely to the user ID $_{i}$.

e) The user $\operatorname{ID}_{i}$ judges the validity of the partial private key by checking $D_{i} P=Y_{i a}+P_{\text {pub }} H_{1}$ (ID $i$ ).

Notably, these procedures finish three different algorithms

which are: 1) set-secret-value; 2) partial-privatekeyextract; and 3) set-public-key of the proposed scheme. These algorithms generate public key $\left(Y_{i b}, Y_{i a}\right)$ that is kept in the public tree by the KGC, and the full private key $\left(x_{i}, D_{i}\right)$ is kept secret by the user.

\subsection{Signcrypt}

This algorithm is performed by a sender $\operatorname{ID}_{i}$ to signcrypt the message $m_{i}$ with $\mathrm{ID}_{R}$ as a receiver. $\mathrm{ID}_{i}$ performs the algorithm as follows.

a) ID $i$ randomly selects $r \in Z_{q}^{*}$ and compute $T_{i}=r P$.

b) Compute $Z_{b}=r Y_{r b}$.

c) Compute $Z_{a}=r\left(Y_{r a}+P_{\text {pub }} Q_{i}\right)$.

d) Compute $h_{a}=H_{2}\left(\operatorname{ID}_{R}\left\|Y_{r a}\right\| Y_{r b}\|\Delta\| T_{i}\left\|Z_{b}\right\| Z_{a}\right)$.

e) Compute $K_{i}=h_{a} \oplus m_{i}$.

f) Compute $h_{b}=H_{3}\left(\operatorname{ID}_{R}\left\|Y_{r a}\right\| Y_{r b}\|\Delta\| T_{i}\left|K_{i} \| Q_{i}\right|\right.$

$\left.\mid Y_{i b} \| Y_{i a}\right)$.

g) Compute $h_{c}=H_{4}(\Delta)$.

h) Compute $\alpha_{i}=D_{i} h_{c}+r h_{b}+x_{i} h_{c}$

i) Return the ciphertext $C_{i}=\left(T_{i}, K_{i}, \alpha_{i}\right)$.

\subsection{Aggregate}

This algorithm is performed by aggregator signcryption generator on the receiver $\mathrm{ID}_{R}$ as follows.

a) Compute $\alpha=\sum_{i=1}^{n} \alpha_{i}$

b) This algorithm outputs the aggregate ciphertexts $C=\left(T_{1} \ldots T_{n}, K_{1} \ldots K_{n}, \alpha\right)$.

\subsection{Aggregate-Verify}

This algorithm is run by a receiver $\mathrm{ID}_{R}$ and computes the following.

a) $h_{b}=H_{3}\left(\operatorname{ID}_{R}\left\|Y_{r a}\right\| Y_{r b}\|\Delta\| T_{i}\left\|Z_{b}^{\prime}\right\| Q_{i}\left\|Y_{i b}\right\| Y_{i a}\right)$, for $i=1, \ldots, n$.

b) $h_{c}=H_{4}(\Delta)$.

c) Verify $\mathrm{e}(\alpha, P)=\hat{\mathrm{e}}\left(\sum_{\mathrm{i}=1}^{\mathrm{n}} Y_{i a}+P_{\mathrm{pub}} Q_{i}, h_{c}\right) \hat{\mathrm{e}}$

$\left(\sum_{i=1}^{n} T i, h_{b}\right) \hat{\mathrm{e}}\left(\sum_{i=1}^{n} Y_{i b}, h_{c}\right)$.

If the above equation holds, this algorithm outputs true otherwise false.

\subsection{Aggregate-Unsigncrypt}

If the output of Aggregate-

Verify algorithm is true, this algorithm is performed by the receiver $\mathrm{ID}_{R}$ as follows.

a) Compute $Z_{b}^{\prime}=x_{r} T_{i}$. 
b) Compute $Z_{a}^{\prime}=D_{r} T_{i}$.

c) Compute $h_{a}^{\prime}=H_{2}\left(\operatorname{ID}_{R}\left\|Y_{r a}\right\| Y_{r b}\|\Delta\| T_{i}\left\|Z_{b}^{\prime}\right\| Z_{a}^{\prime}\right)$.

d) Compute $m_{i}^{\prime}=K_{i} \oplus h_{a}^{\prime}$.

e) This algorithm outputs $\{m i\}_{i=1}^{n}$.

\subsection{Correctness of the Signatures}

$$
\begin{aligned}
& \hat{\mathrm{e}}(\alpha, \mathrm{P})= \hat{\mathrm{e}}\left(\sum_{i=1}^{n} \alpha_{i}, P\right) \\
&= \hat{\mathrm{e}}\left(\sum_{i=1}^{n}\left(D_{i} h_{c}+r h_{b}+x_{i} h_{c}\right), P\right) \\
&=\hat{\mathrm{e}}\left(\sum_{i=1}^{n} D_{i} h_{c}, P\right) \hat{\mathrm{e}}\left(\sum_{\mathrm{i}=1}^{\mathrm{n}} r P, h_{b}\right) \hat{\mathrm{e}}\left(\sum_{\mathrm{i}=1}^{\mathrm{n}} x_{i} P, h_{c}\right) \\
&=\hat{\mathrm{e}}\left(\sum_{i=1}^{n} D_{i} P, h_{c}\right) \hat{\mathrm{e}}\left(\sum_{\mathrm{i}=1}^{\mathrm{n}} T_{i}, h_{b}\right) \hat{\mathrm{e}}\left(\sum_{\mathrm{i}=1}^{\mathrm{n}} Y_{i b}, h_{c}\right) \\
&=\hat{\mathrm{e}}\left(\sum_{i=1}^{n}\left(Y_{i a}+P_{\mathrm{pub}} Q_{i}, h_{c}\right)\right) \\
& \quad \times \hat{\mathrm{e}}\left(\sum_{\mathrm{i}=1}^{\mathrm{n}} T i, h_{b}\right) \hat{\mathrm{e}}\left(\sum_{\mathrm{i}=1}^{\mathrm{n}} Y_{i b}, h_{c}\right)
\end{aligned}
$$

\subsection{Correctness of the Decryption}

$$
\begin{aligned}
m_{i}^{\prime} & =K_{i} \oplus h_{a}^{\prime} \\
& =H_{2}\left(Q_{i}\left\|Y_{i a}\right\| Y_{i b}\|\Delta\| T i\left\|Z_{b}\right\| Z_{a}\right) \oplus m_{i} \oplus h_{a}^{\prime} \\
& =h_{a} \oplus m_{i} \oplus h_{a}^{\prime} \\
& =m_{i} .
\end{aligned}
$$

\section{RESULTS AND DISCUSSION}

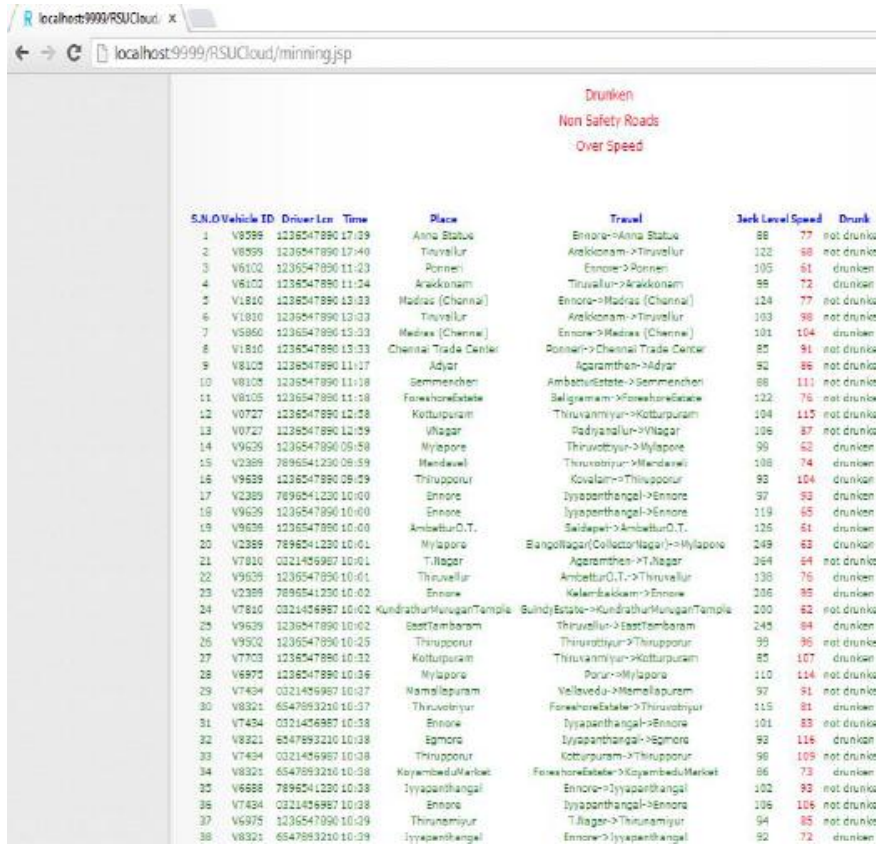

Fig.3: Vehicle data set

Fig 3 shows the data set of the vehicles. This data set contains the sensed information such as Jerk Level, Speed and Safety between the source and destination. These information are useful for the mobile users who requests the safe route. Fig 4 shows the best and safe route between source and destination and additionally it displays the Public Transport for the requested route.

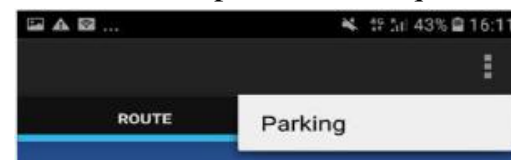

\section{Path Inforamation}

Vadapalani->KKNagar->Saidapet->Adyar$>$ AvvaiHome->BesantNagar

\section{Public Transport}

\section{$5 E$}

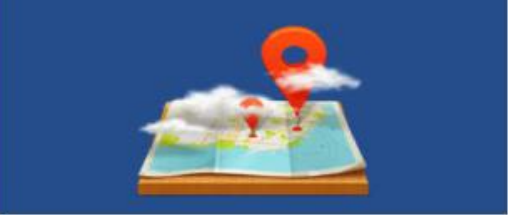

Fig.4: Dipslaying Best Route

Fig 4 displays the best route in map. It points the best intermediate route between source and destination. As it points the places, it will be very comfortable for the mobile users to travel easily and safely.

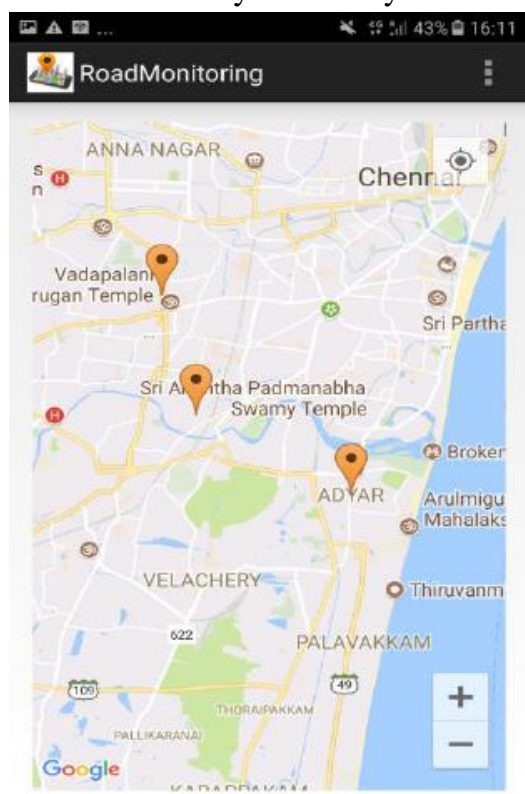

Fig.5: Route in map

Fig 5 displays the additional information to the mobile users such as the number of restaurants, petrol bunks, hospitals. 


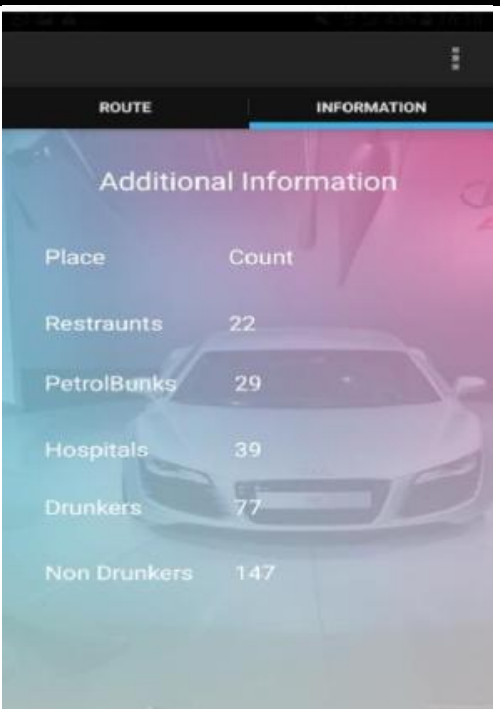

Fig.6: Additional Information

\section{CONCLUSION}

We propose a new efficient CLASC scheme. We then designed a privacy preserving vehicular crowd sensing Road Surface Condition monitoring system using fog computing based on the proposed CLASC scheme. In addition, the proposed privacy-preserving protocol meets the security requirements such as data confidentiality and integrity, mutual authentication, anonymity, and key escrow resilience. Extensive comparisons of computational cost and communication overhead show that the proposed scheme can achieve much better efficiency than the existing schemes.

\section{REFERENCES}

[1] M. Scott. Efficient Implementation of Crytographic Pairings. Accessed on Feb. 18, 2017. [Online]. Available: http://ecryptss07.rhul.ac.uk/Slides/Thursday/mscott-samos07.pdf

[2] Winter Driving-Be Prepared, Be Safe, Ontario Ministry Transp.,Toronto, ON, Canada, Feb. 2017. [Online].

Available: http://www.mto.gov.on.ca/english/ontario511/pdfs/winter-safe-driving.pdf

[3] J. Kar, "Provably secure identity-based aggregate signcryption schemein random oracles," Int. J. Netw. Security, vol. 17 , no. 5, pp. 580-587,Sep. 2015

[4] L. M. Vaquero and L. Rodero-Merino, "Finding your way in thefog: Towards a comprehensive definition of fog computing," ACMSIGCOMM Comput. Commun. Rev., vol. 44, no. 5, pp. 27-32, 2014

[5] Z. Eslami and N. Pakniat, "Certificateless aggregate signcryption:Security model and a concrete construction secure in the random oraclemodel," J. Comput. Inf. Sci., vol. 26, no. 3, pp. 276-286, 2014.

[6] S.-H. Seo, M. Nabeel, X. Ding, and E. Bertino, "An efficient certificateless encryption for secure data sharing in public clouds," IEEE Trans.Knowl. Data Eng., vol. 26, no. 9, pp. 2107-2119, Sep. 2014.

[7] I. Stojmenovic, "Fog computing: A cloud to the ground supportfor smart things and machine-tomachine networks," in Proc. Aust.Telecommun. Netw. Appl. Conf. (ATNAC), Southbank, VIC, Australia,2014, pp. 117-122.

[8] N. Mitton, S. Papavassiliou, A. Puliafito, and K. S. Trivedi, "Combiningcloud and sensors in a smart city environment," EURASIP J. WirelessCommun. Netw., vol. 2012, p. 247, Dec. 2012.

[9] M. Perttunen et al., "Distributed road surface condition monitoring usingmobile phones," in Ubiquitous Intelligence and Computing. Heidelberg,Germany: Springer, 2011, pp. 64-78.

[10] G. Strazdins, A. Mednis, G. Kanonirs, R. Zviedris, and L. Selavo,"Towards vehicular sensor networks with android smartphones for roadsurface monitoring," in Proc. 2nd Int. Workshop Netw. CooperatingObjects (CONET) Electron. CPS Week, Chicago, IL, USA, 2011,pp. 1-4.

[11]W. Xie and Z. Zhang, "Efficient and provably secure certificateless signcryption from bilinear maps," in Proc. IEEE Int. Conf.Wireless Commun. Netw. Inf. Security (WCNIS), Beijing, China, 2010,pp. 558-562.

[12]F. Li, M. Shirase, and T. Takagi, "Certificateless hybrid signcryption," in Proc. 5th Inf. Security Pract. Experience Conf. (ISPEC), vol. 5451.Xi'an, China, 2009, pp. 112-123.

[13] C. Wu and Z. Chen, "A new efficient certificateless signcryption scheme," in Proc. Int. Symp. Inf. Sci. Eng. (ISISE), vol. 1. Shanghai,China, 2008, pp. 661-664.

[14] A. W. Dent, "A survey of certificateless encryption schemes and security models," Int. J. Inf. Security, vol. 7, no. 5, pp. 349-377, 2008.

[15]B. C. Hu, D. S. Wong, Z. Zhang, and X. Deng, "Certificateless signature:A new security model and an improved generic construction," Design Codes Cryptography, vol. 42, no. 2, pp. 109-126, 2007. 